\title{
Interoperability of disparate engineering domain ontologies using Basic Formal Ontology
}

Thomas J. Hagedorn, Barry Smith, Sundar Krishnamurty \& Ian Grosse

\begin{abstract}
As engineering applications require management of ever larger volumes of data, ontologies offer the potential to capture, manage, and augment data with the capability for automated reasoning and semantic querying. Unfortunately, considerable barriers hinder wider deployment of ontologies in engineering. Key among these is lack of a shared top-level ontology to unify and organize disparate aspects of the field and coordinate co-development of orthogonal ontologies. As a result, many engineering ontologies are limited to their scope, and functionally difficult to extend or interoperate with other engineering ontologies. This paper demonstrates how the use of a top-level ontology, specifically the Basic Formal Ontology (BFO), greatly facilitates interoperability of multiple engineering-related ontologies. We constructed a system of formal linked ontologies by re-engineering legacy ontologies to be conformant with BFO and developing new BFO-conformant ontologies to capture knowledge in the engineering design, enterprise, human factors, manufacturing, and application domain of additive manufacturing. The resulting Integrated Framework for Additively Manufactured Products (IFAMP), including the body knowledge instantiated on its basis, serve as the basis for a proposed Design with Additive Manufacturing Method (DAMM), which we believe can support the design of innovative products with semantically enhanced ideation tools and enhanced access to application domain knowledge. The method and its facilitation through the ontological framework are demonstrated using a case study in medicine.
\end{abstract}




\section{Introduction}

\subsection{Additive Manufacturing}

\subsubsection{Overview of Additive Manufacturing}

Additive manufacturing (AM) comprises a range of manufacturing technologies that construct parts by progressively adding material, as opposed to removing material, which is what happens in conventional machining. Compared to additive processes such as welding, AM typically employs an additive process that is being driven by a computer working to replicate the geometry specified in a digital model. The primary mechanisms and methods for additive manufacturing using stereolithography techniques were first proposed in a series of patents in the 1950s (Bourell et al. 2009). Since its inception, additive manufacturing has expanded to support an increasing set of methods. These include processes to fabricate parts from a variety of plastics, ceramics, metals, and even biological materials. The technology has moreover seen expanding use in areas as diverse as rapid prototyping, mass customization of products, aerospace, and medical devices (Wohlers and Gornet 2011).

The main advantages of AM come from its low tooling costs, which allow cheap customization, and from its ability to create parts with high complexity. Gibson et al. (Gibson et al. 2010), for example, note that AM can manufacture structures that exhibit not only shape but also functional and hierarchical complexity. Shape complexity refers to the intricate geometries that are possible with AM. Functional complexity is the ability of a single product to do many things and to consolidate assembly functions into a single part. Hierarchical complexity describes the inclusion of both macro and mesoscale features. On the other hand, however, these advantages come with significant costs. These include costs of materials (Ruffo, Tuck, and Hague 2006), availability of common engineering materials (Bourell et al. 2009; Kumar and Kruth 2008), performance costs (Barclift and Williams 2012; Kumar and Kruth 2008), as well as slow process speeds and scalability issues.

\subsubsection{Design for Additive Manufacturing}

Such concerns, combined with the creative challenge introduced by significantly greater design freedom, have led to the development of novel methods tailored specifically for design for additive manufacturing (DFAM). These include methods to help designers take advantage of greatly expanded design freedom which AM affords. A common DFAM approach is to focus on

process limitations. Such methods include topological optimization around manufacturing 
constraints (Gardan and Schneider 2015), single-process design checking approaches, and generalized design guidelines for additive manufacturing (Gibson et al. 2010; Perez, Anderson, and Wood 2015; Thomas 2009; Adam and Zimmer 2014). Many methods focus on the creative aspects of design. These include approaches encouraging functional consolidation of assemblies into single parts (Yang and Zhao 2015), on-line design repositories depicting AM solutions (Laverne et al. 2015; Rodrigue and Rivette 2010), tables of useful features (Bin Maidin, Campbell, and Pei 2012), and team-based methods integrating AM expertise early in the design (Laverne et al. 2015). Unfortunately, however, these methods typically deal with just one single type of AM process, and they prove difficult to generalize to other cases. Alternatively, they consider AM as a monolithic technology rather than as a diverse set of related technologies having significantly different capabilities and applications. They thus ignore many of the knowledge-based challenges associated with AM, or they artificially restrict the design space so as to mitigate them.

The sheer scope and complexity of the sorts of knowledge, data and tools required for DFAM have spurred interest in knowledge management approaches for AM. For example, one such framework used a simple semantic network to link AM product designs to a limited set of “values” that they might possess (Kumke, Watschke, and Vietor 2016; Kumke et al. 2017). While a valuable approach, the simplicity of a simple network likely limits its ability to capture detailed product information and support complex or highly specific queries addressed against the available data. Other work has focused on using ontologies as a tool to support DFAM itself. These include the creation of an ontology of additive manufacturing processes (Eddy et al. 2015) and a design for additive manufacturing ontology (Dinar and Rosen 2017). More recently a modular multidomain ontology was used to link process and machine capability information to a repository of AM designs, which was then used to construct a set of sample queries to aid in design (Hagedorn, Krishnamurty, and Grosse 2018). While we believe that these represent significant contributions in ontology development for additive manufacturing, it is notable that neither of these resources incorporated robust methods for incorporating into the design process knowledge from the product domain, for example knowledge about properties of materials or about product use environments. This represents a major shortfall, as such knowledge would both allow better decision-making regarding design choices and assist in the development of more innovative products. 


\subsubsection{Additive Manufacturing in Medicine}

AM has seen an increasing number of applications in medicine. Common applications include anatomical models for training (Waran et al. 2014), planning and education through use of 3D digital models (Preece et al. 2013), cutting and drilling guides customized to patient anatomy (Barrack et al. 2012; Slover et al. 2012; Nam et al. 2013), and creating orthopedic prostheses similarly customized to a specific patient (Lethaus et al. 2012; Farré-Guasch et al. 2015; Jardini et al. 2014; Dérand, Rännar, and Hirsch 2012). Despite its promise, however, many issues prevent wider adoption of AM in medicine. These range from concerns over safety, reliability, and accuracy of AM parts on the one hand to logistical problems on the other. There are also broader concerns over how AM might be used economically for medical applications (Martelli et al. 2016). Even absent the added considerations of DFAM, medical environments can be challenging for designers due to factors such as limited accessibility of domain knowledge (Martin et al. 2008; Demers-Payette, Lehoux, and Daudelin 2016; Nagel et al. 2008), restricted access to stakeholders (Martin et al. 2008), pervasive regulatory concerns (Kaplan et al. 2004), complicated market structures (Aquino Shluzas, Steinert, and Leifer 2011), and overall difficulty in assessing the requirements and value of new technologies (Martin et al. 2012). Like DFAM generally, therefore, designing for additive manufacturing in medicine requires the application of domain specific knowledge to aid in design, decision making, and ultimately innovation. Unfortunately, medical device design methods rarely offer much in the way of guidance or software tools for applying medical domain knowledge. One potential solution is knowledge management through ontologies, a solution that is all the more attractive given the large body of sophisticated biomedical ontology resources, not least in the domain of anatomy (Rosse and Mejino 2008).

\subsubsection{Ontology Development}

Ontologies are a potentially powerful tool for knowledge management (Chandrasegaran et al. 2013). An ontology represents domain knowledge in the form of a hierarchy of terms describing some domain, relations between these terms, and definitions that may be used to classify specific entities (Gruber 1993; Gruber 1995). The result is a machine and human readable representation of entities and relations that may be used to annotate (or tag) otherwise ambiguous information, provide it with context, promote interoperability with information from other sources or domains, and reason over the information using automated reasoning software (Gruber 1991). This formalization of knowledge in a machine-readable format is key to the implementation of the 
semantic web and semantic web tools, which seek to annotate and organize data retrieved from online repositories using controlled terminologies to provide highly sophisticated data-based services (Berners-Lee, Hendler, and Lassila 2001).

There are two main types of ontology: top-level ontologies (TLOs) and domain ontologies. TLOs provide a high-level philosophical view of the world, which alongside development aids such as style guidelines and additional documentation may be used to develop more specialized domain ontologies ((Breuker, Valente, and Winkels 2004; Fox and Gruninger 1998; Cowell and Smith 2010)). TLOs are formulated so as to be maximally general and are such that their definitions do not presuppose the use of any more general terms at higher levels. Examples of TLO terms are 'object', 'process', 'spatial region', and so forth. Domain ontologies by contrast are ontologies that are developed to represent the knowledge and terminology specific to some professional, scientific, or similar domain. Ideally domain ontology terms are defined in such a way that they draw on one or more terms of the sort included in top-level ontologies. Examples of domain ontology terms are 'machine’, ‘welding', 'porcelain'.

As Gruber saw already in 1993, provided these domain ontologies adhere to shared development principles and do not overlap by replicating each other's terminology, ontologies are naturally interoperable with one another, shareable, and extensible to new domains (Gruber, 1993). Combined with infrastructure to share, vet, and curate new ontologies the use of a common toplevel ontology makes it possible to coordinate powerful co-development efforts by drawing on shared principles and expertise. It also provides the computational basis for consistent application of semantic web tools across multiple fields. In biomedicine, for example, efforts like the Open Biomedical Ontologies (OBO) Foundry (Smith et al. 2007) and the Human Phenotype Ontology) (Le, Dao 2018, Robinson 2012) have shown the power of sophisticated domain ontologies to advance clinical science through deployment of semantic web tools.

\subsubsection{Ontologies in Engineering}

As with the specific case of AM, ontologies have been touted as a potential solution for the explosion in complexity of data and knowledge used in intensive engineering design projects generally. Past research has led to the development of many ontologies for engineering domains and subdomains. These include multiple proposed upper level ontologies (Saeema and Mario 2007; Štorga, Andreasen, and Marjanović 2010), terminologies to support modeling of functions (Hirtz et al. 2002) and of products (Fenves 2001), as well as ontological representations of such 
terminologies (Fernandes et al. 2007; Eddy et al. 2011), and ontological approaches for tracking information relating to manufacturing capabilities (Ameri and Dutta 2006) among many others. Other work has focused ontologies describing different aspects of engineering design, modeling and innovation, including ontologies aiming to capture data relating to engineering models (Grosse, Milton-Benoit, and Wileden 2005), decision making (Rockwell et al. 2009), usable design (Hagedorn, Krishnamurty, and Grosse 2016), laminated composite materials (Premkumar et al. 2014), sustainable design (Eddy et al. 2014), as well as a modular ontological framework for design (Eddy et al. 2011) and multidisciplinary work using ontologies for annotating bio-models (McPherson et al. 2013) and for medical device innovation (Hagedorn, Grosse, and Krishnamurty 2015).

Despite the breadth of ontologies proposed for various engineering subdomains, however, their use in industry remains relatively rare. While several factors are likely culprits in this lack of uptake, many issues stem from failures of interoperability. There are just too many engineering ontologies, almost all of which are developed in an ad hoc fashion with little attention to issues of orthogonality, cross-ontology compatibility and sustainability. Few engineering ontologies utilize a top-level ontology to organize their terms, provide development guidelines, or establish a basic philosophical-architectural perspective. As a result, few engineering ontologies adhere to shared modeling principles, and so interoperability between any two engineering ontologies is rare. There has been relatively little co-development or -curation of engineering ontologies, which stands in stark contrast to fields such as biomedicine that have seen widespread collaborative development and use of ontologies and ontology-based tools. Many ontologies published in the engineering literature have not been made publicly available, meaning they provide little in the way of input to subsequent ontology development or of lessons of consequence for the construction and application of ontology-based engineering tools by subsequent generations. Within the field of engineering - again as contrasted with biomedicine (Noy et al. 2009) - curated ontology repositories are rare. As a result, efforts to develop new, high quality engineering ontologies and semantic web applications must often start from scratch.

Existing engineering ontologies are often overlapping, non-interoperable, unreadable by humans, and defined in an esoteric fashion that limits their usefulness to the broader community. For simple, self-contained applications these issues may not be significant. However, few engineering applications are simple, and many of the core advantages offered by ontologies depend 
precisely on formality, interoperability, availability, and usefulness beyond any single application. More recent efforts seek to address these issues with coordinate co-development of engineering domain ontologies (Mohd, Rai, and Otte, 2019; Wallace, Kiritsis, and Smith 2018), but at present there are few demonstrations of highly interoperable engineering ontologies

\subsubsection{Objectives and Scope}

This paper has two main objectives. First, we will seek to demonstrate the utility, power, and potential of ontologies developed using not only a shared top-level ontology but also shared development principles and modular, orthogonal structure. It will aim to do so by showcasing the development of an ontological framework combining prior work in the additive manufacturing domain developed using these principles with broader knowledge reflecting stakeholders. This framework is constructed so that it may be extended with knowledge from specific problem domains. This approach is novel as it requires both more typical domain ontology development and suite level semantic considerations to permit extension to new domains While we will focus here on the use of AM in the medical field, the ontology is formulated so that it might be applied to virtually any engineering design problem and incorporate existing ontologies as needed. Second, this work applies the proposed ontology suite to advance a novel new engineering design method. The method serves to demonstrate how ontological framework may facilitate the design of innovative products based on knowledge intensive, automated design checking and query-based design ideation.

\section{Methods}

\subsection{Development of Ontology}

The ontological framework developed for this work is designed to provide an Integrated Framework for Additively Manufactured Products (IFAMP). It is envisioned that such a unified framework will allow useful cross-domain reasoning that will facilitate the development of more innovative designs in fields such as those depicted in Figure 1. It provides an ontological representation of the design, intended manufacturing processes, usability, and innovation that may be used to facilitate rule-based semantic reasoning and a knowledge base accessible through queries. IFAMP is also intended to serve as a demonstration of how ontology development methodologies such as adoption of a shared TLO and development principles, modularity, orthogonality, and reuse of existing ontologies might help reduce or eliminate barriers to wider adoption in industry. 


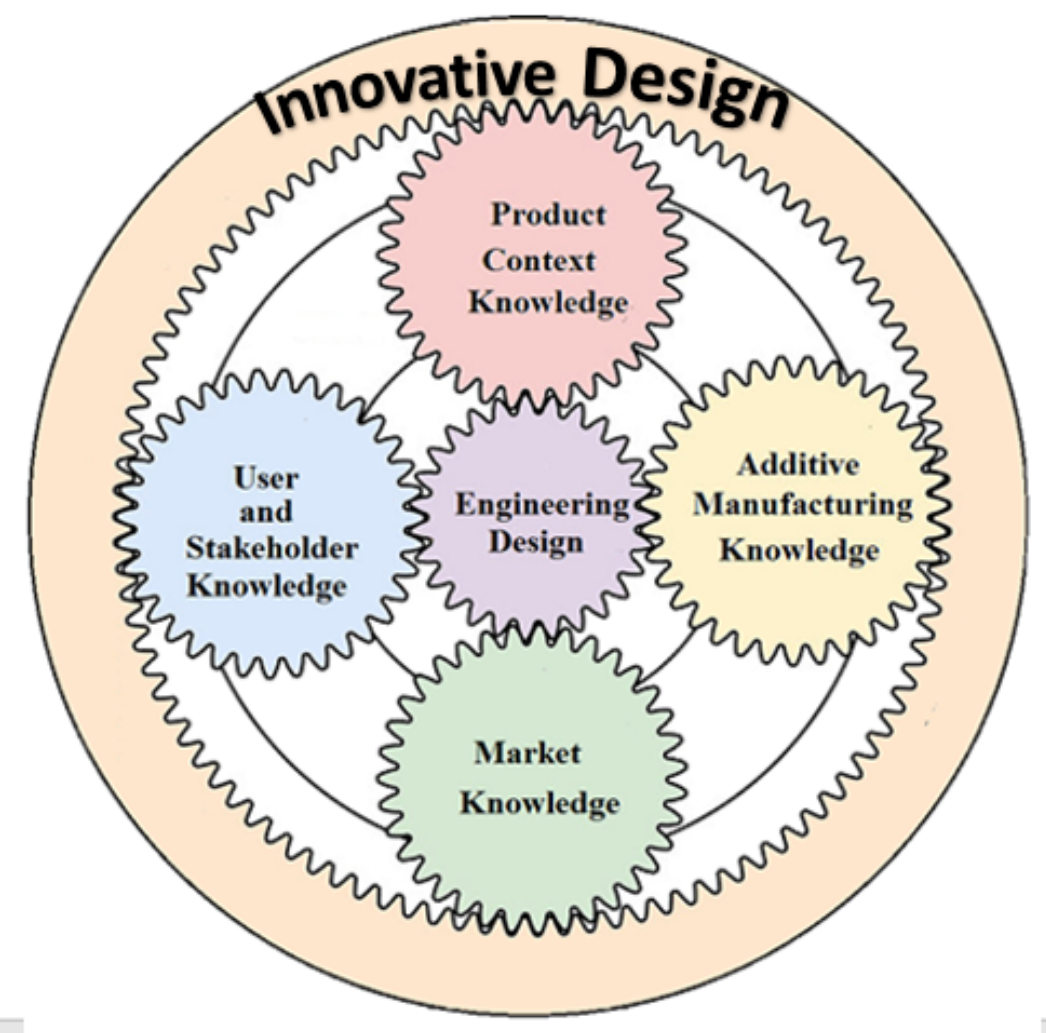

Figure 1. Overview of the unified design framework implemented in IFAMP. Gear colors will be used to indicate knowledge domains in subsequent figures.

While this work primarily focuses on a medical application area, knowledge of virtually any field might be substituted for the medicine-specific aspects of this work. IFAMP was developed using a highly modular structure so as to support the ability to substitute or expand specific domain knowledge without needing to revise or even substantially modify the rest of the ontology suite. Every domain ontology comprising IFAMP may be separated from the greater whole and incorporated into new ontologies or applications.

\subsubsection{Top-Level Ontology}

IFAMP was implemented under a shared top-level ontology and makes extensive use of past work published in the literature. The selected TLO was used to help proscribe a basic view of the world for the IFAMP framework, allowing easier rationalization of the included knowledge domains. The Basic Formal Ontology (BFO) (Arp, Smith, and Spear 2015) was selected for this work for several reasons: 
1. The existence of several well vetted mid-level ontologies defined in its terms (Smith and Ceusters 2015; Smith et al. 2005),

2. The availability of high-quality documentation and style guidelines (Arp, Smith, and Spear 2015),

3. Complementary prior and ongoing work using BFO (Furini et al. 2016, Mohd, Rai, and Otte, 2019; Wallace, Kiritsis, and Smith 2018)

Since ontologies sharing a common top-level and adhering to the same development principles can often be linked seamlessly with one another, use of BFO allowed multiple domain ontologies to coexist and interoperate more easily. In this work BFO allows IFAMP repurpose previously reported ontologies with little to no additional work. It also means that subsequent development efforts can do the same with the IFAMP ontologies. This allows more rapid and less labor-intensive development of ontologies for new domains.

Within BFO all entities are split into two disjoint groups dubbed Continuants and Occurrents respectively. Continuants can be thought of as entities described via a sequence of snapshots in time, such as people, traits such as temperature or mass, and information entities such as documents or databases. Continuants maintain their identity from one snapshot - which means from one moment in time - to the next. There are three distinct subtypes of continuant. Independent continuants, which exist independently of other entities. This class includes things like objects, environments, natural engineered systems, spatial regions, and the like. Specifically, dependent continuants by contrast must be borne by other entities. Things such as shape, mass, and function for example are specifically dependent on some independent entity having geometry, mass, or function. Generically dependent continuants similarly depend for their existence on some other entity but may be borne by different entities at different times, or by many entities at the same time. This includes things like digital records and other information content entities, including designs and software. Occurrents, in contrast, unfold in time and include things like processes, lives, histories, and processing realizing the functions of artifacts such as the pumping processes realizing the function of a pump. (Arp, Smith, and Spear 2015).

\subsubsection{Use of Existing BFO Conformant Ontologies}

Several existing BFO conformant ontologies were used to facilitate the development of IFAMP. The Common Core Ontologies ${ }^{1}$ were used as a mid-level between BFO and more specific

\footnotetext{
${ }^{1}$ https://www.cubrc.org/index.php/data-science-and-information-fusion/ontology
} 
domain ontologies. The Common Core is a suite of ontologies proposed as a high-level terminology resource to support the development of BFO conformant ontologies. It provides terms for dealing with data and information relating to various types of person, time, spatial position, and qualities of entities. This terminology advances a set of triple patterns that may be refined and extended to formulate new domain ontologies. This repetition of patterns allows more straightforward semantic reasoning and easier querying because data mapped to the ontology is treated in a consistent manner across domains and often uses shared super-properties. The Common Core's contents are largely developed either by borrowing from existing OBO ontologies or by developing new ontologies but according to the same principles (Smith et al. 2007). Given their relatively abstract terminology, nesting the IFAMP ontology within BFO and the Common Core provides opportunities to interoperate with ontologies describing many domains.

Other domain ontologies were also used to describe the knowledge domains covered by IFAMP. As the creation of a design repository to aid in design ideation is central to its envisioned use, IFAMP draws upon the Innovative Capabilities of Additive Manufacturing (ICAM) ontology (Hagedorn, Krishnamurty, and Grosse 2018), which implements ontology-linked knowledge bases focusing on innovative product design. The ICAM ontology provides content representing the manufacturing, value, and innovation domains in our new framework. ICAM includes suitably modified versions of the Manufacturing Service Description Language (MSDL) (Ameri and Dutta 2006), the Semantic Additive Manufacturing Process Ontology (SAMPro) (Eddy et al. 2015), and the Functional Basis Ontology (FBO) (Fernandes et al. 2007). Taken together, these ontologies include an ontological model of the manufacturing domain, additive manufacturing processes, and of functional models.

\subsubsection{Development of a Human Factors Ontology}

In addition, a high-level human factors ontology was included in order to capture information about the users of a device and associated stakeholders such as clinicians, hospital regulators, and so forth. Though independently developed for this work, its core functionality is based on previous work using ontologies to link design and user information (Hagedorn, Krishnamurty, and Grosse 2016). As this prior work was developed without a top-level ontology however, it had to be re-developed using the BFO structure in order to be attachable to a broader

ontological framework. This re-engineering process also served to expand the extent and quality of information about device users that could be captured. The human factors ontology aims to 
define terms relating not only to stakeholders in a design process such as end users, but also their relevant capabilities, and their preferences towards some aspect of a design. While device users are the primary focus, regulators, and by extension regulations and standards were also included, as these are of particular importance in the medical domain (Figure 2).

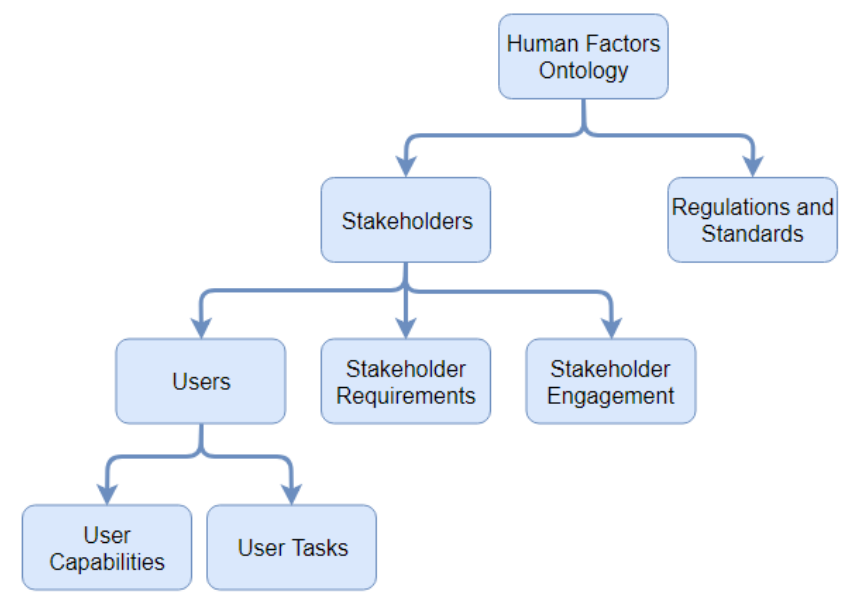

Figure 2. Information Domains Captured by the Human Factors Ontology

\subsubsection{Regulatory Terms}

The regulatory portion of the human factors ontology defines the classes needed to capture regulatory information. Various aspects of regulations and standards are treated as types of directive information entities or designative information content entities as defined in the Information Artifact Ontology (IAO) (Smith and Ceusters 2015) and Common Core. A similar treatment is given to standards that may have application in some given product domain. Each regulation has some scope of entities that it affects, which is defined via a has within scope object property. This property points from specific regulations to, for example, a specific class of medical devices indicated by a regulatory classification. A regulated device has classification some regulatory classification. A has consequence property in points from regulatory classes to design requirements that might result from being classified by some regulator (Figure 3). 


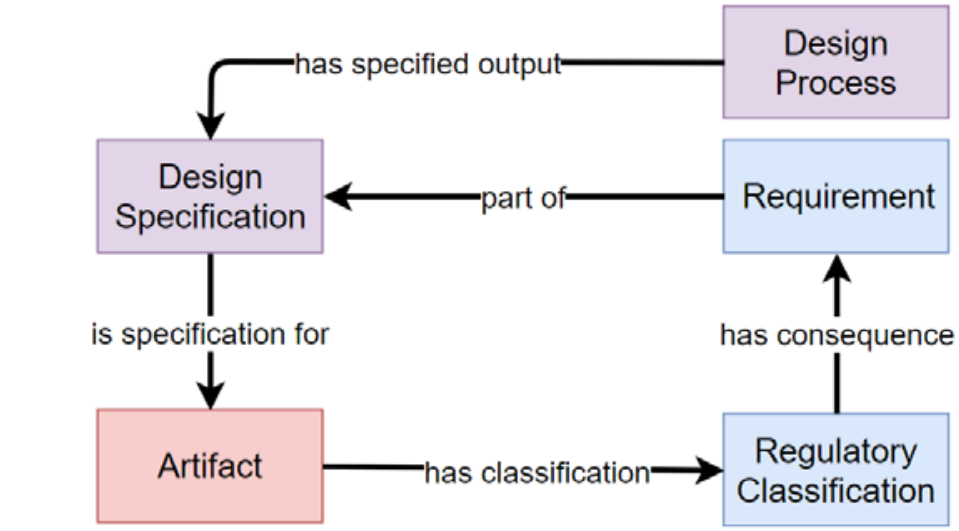

is specification for o has classification o within scope of o has consequence

$\rightarrow$ has part

Figure 3. Information model and axioms linking regulatory requirements to a design Using a property chain (bottom), one can infer that specifications for an artifact falling within the scope of a regulation will inherit certain consequences (requirements) from the regulation.

The choice of representing various types identified by specific regulations deliberately aims to avoid over-committing the ontology to one specific viewpoint (for example that of the FDA regulator, or that of a specific hospital), and thereby limit IFAMP's formality, modularity, and extensibility. It is also intended to keep the ontology consistent with BFO recommendations to avoid multiple inheritance wherever possible (Arp et al. 2015). As structured, the ontology only asserts that some entity (a regulation) uses some terminology and its associated definitions to classify some entity. The associated classifications need then be neither unique nor definitive. For example, regulators from different nations might maintain different classifications for a given type of medical device, each with different impacts on its design. At the same time, the link between actual devices, regulatory classes (a designation applied to devices), and regulations simply extends and makes more specific existing triple patterns established in the Common Core. Understanding the more general semantics thus allows rapid interpretation of the specific case of device regulations.

Each regulation has consequences affecting anything - whether an object or a process that falls within its prescribed scope. An entity that falls within the scope of the regulation must then bear certain consequences, typically in the form of additional requirements. These consequences will then affect both the classified thing and any specification that describes it. For 
example, a design of a medical device, for a device that falls within the scope of a US medical device regulation, instances representing the requirements imposed by that regulation would be automatically linked via a part of relation from the Common Core (as in Figure 3). These in turn are about various processes, methods, and the like which must be considered in a design or a design process.

Specific regulatory classes and instances were added to describe FDA regulations pertaining to medical devices. On top of this, the medical device terminology was enriched with additional information about risk and regulatory classes. To take account of the fact that any object can have an arbitrary number of regulatory classifications assigned to it, FDA risk classes and medical device classifications coming from any given medical classification system or ontology can coexist with one another within the IFAMP framework without introducing multiple inheritance issues.

\subsubsection{Stakeholder Ontologies}

The stakeholder portions of the Human Factors Ontology (HFO) capture information relating to the needs of users of devices or any other stakeholder, as well as the processes by which these needs are elicited. The User Capabilities Ontology (UCO) deals with both the mental and physical capabilities of device users and with the capabilities demanded to perform some devicerelated process. These are then combined with the regulatory model to form an overall ontology for dealing with human factors components of a design. This combined model additionally captures the types of process involved when a user realizes one or other set of capabilities. A stakeholder figures in the ontology according to their role - as user, owner, customer, etc. - in relation to the product described in a design.

The ontology thus takes account of the fact that (in the medical device domain) a person (or more likely a group of persons) is being directly considered in the design process. Their existence might be inferred when the realization of a design in the form of a product is then used in some process that the user performs. Inferences of this sort are supported by the ontology (Figure 4). 


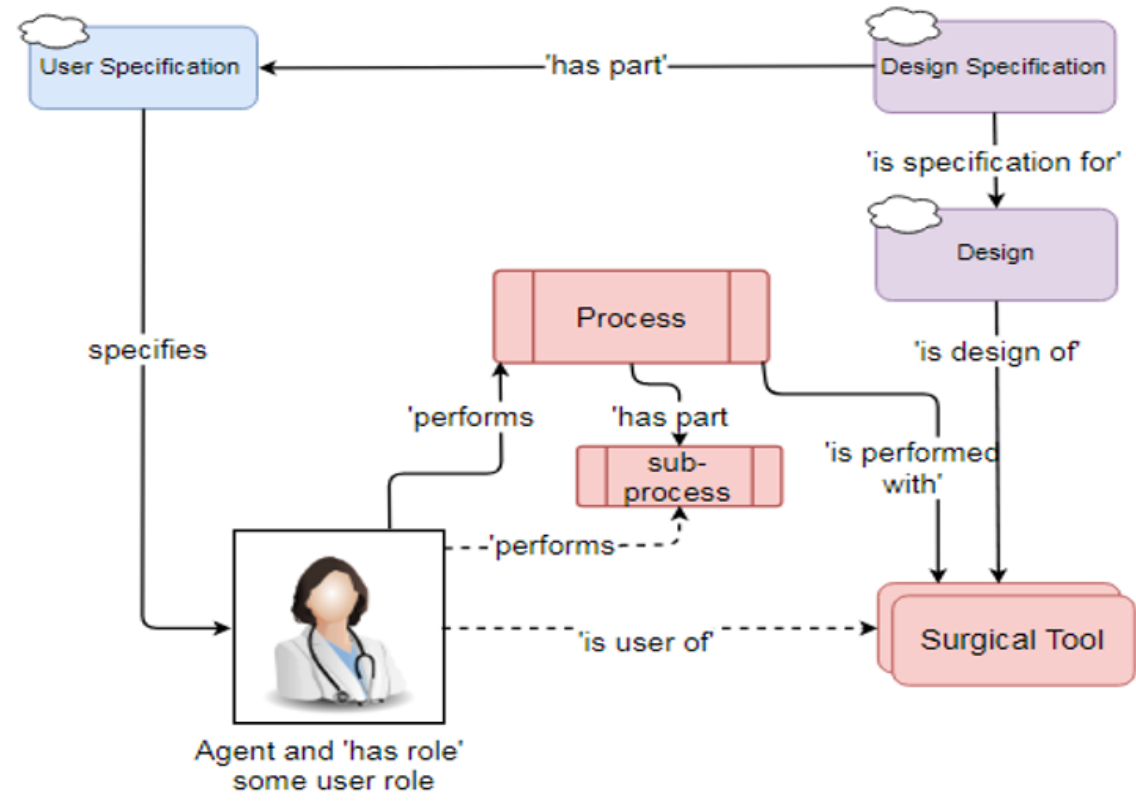

Figure 4. User processes and their relation to a design Solid lines indicate asserted relations.

Dotted lines indicate inferred relations.

Capabilities are treated as dispositions that are borne by continuants. For the purposes of the ontology, a capability is simply defined as a beneficial disposition of some continuant to successfully be able to participate in a process in some pre-specified way. This implies not just participation, but some quality of participation. Thus, the state of bearing capabilities enables successful participation in various processes.

The human factors ontology extends characterization of capabilities to those relevant to the usability of a product. Within the context of a proposed design, users have some set of tasks (processes that they must participate in) that are required for them to successfully use the designed artifact in the manner the designer intends (Figure 5). 


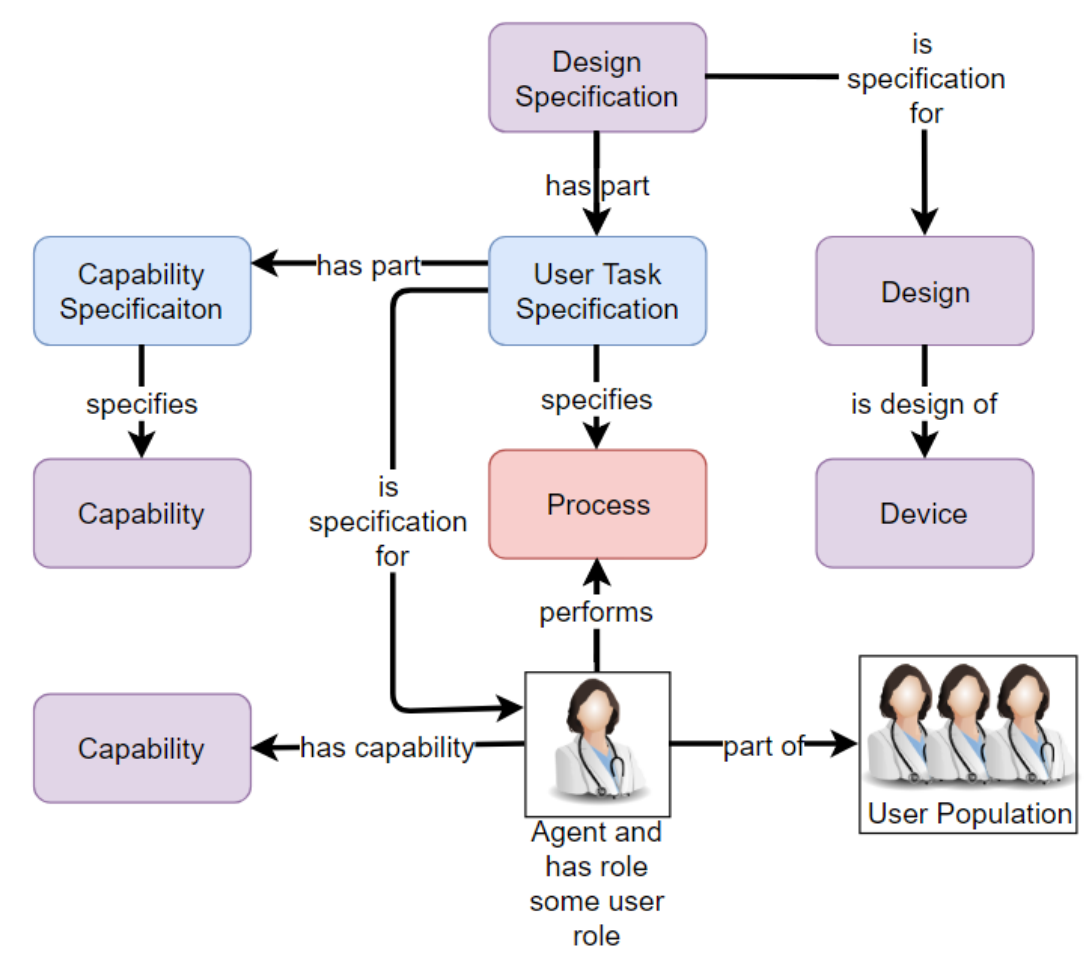

Figure 5. Information model describing user capabilities enabled by the ontology.

Capturing this information and the user's related capabilities allows for potential comparisons between the user capability and the specified capability requirements. For example:

Rule: swrlb:lessThan(?uv, ?sv), specifies(?creq, ?cap), 'has disposition'(?entity,

?cap), 'has specified value'(?cap, ?uv), 'has specified requirement'(?entity,

?creq), 'specifies minimum value'(?creq, ?sv) ---> 'has problem'(?entity, ?creq) ${ }^{2}$

The above rule simply compares a specified value of required performance and the actual performance within the scope of that specification and flags a mismatch as a problem.

As the involved terms are defined under a more universal definition, this rule need not be limited to evaluating usability issues. For example, it might alternatively be used to evaluate disparities between the capabilities of a manufacturing system and those required by some design. More specialized data properties can be used to formulate various types of usability issues, such as ranges of acceptable capabilities. One might for example infer usability assessments from instantiated knowledge of capabilities of various user populations (Hagedorn, Krishnamurty, and Grosse 2016, 181-194). Since capabilities are defined identically across ontologies, an ontology

\footnotetext{
${ }^{2}$ Simplified: If an entity has some requirement, and said requirement specifies some trait be at some level, then if the actual level of the trait is less than the requirement in question is a problem for the entity.
} 
reasoner can, using a very limited rule set, check virtually any capability of any type against requirement specifications related to the design. This includes also capabilities of machines, for example those used in additive manufacturing, where it will allow use of ontologies in assessing machine-level feature fabrication capabilities. In the newly defined human factors ontology, it allows the reasoner to assess the usability of manufactured devices. In the simplest case, the results of such assessment may take the form of a binary decision - as in the rule above. One could however implement population style assessments of the sort described in (Hagedorn, Krishnamurty, and Grosse 2016, 181-194).

Domain-specific rules relating to assessment of usability were added to the ontology as a separable ontology file. This allows the medically specialized aspects of IFAMP to be removed for application in other domains. A similar approach is used throughout IFAMP for the same reason. A set of 15 rules previously developed for medical device design (Ginsburg 2005, 213219) was instantiated in the human factors ontology to support design of medical devices with specific usability features. While the rules themselves cannot be assessed automatically, assessment can be performed by a designer, and violations populated within the ontology. Once populated, usability rules use the same information model as other types of rule that might be used to expand IFAMP, such as manufacturing rules.

\subsubsection{Introduction of Medical Terminology}

\subsubsection{Selection of a Clinical Information Model}

Terminology reflecting medical devices and environments were included to specialize the implementation of IFAMP presented in this paper for the development of medical tools. While a previous effort at using ontologies to support medical device innovation (Hagedorn, Grosse, and Krishnamurty 2015, 218-230) used the Systemized Nomenclature of Medicine Clinical Terminology (SNOMED CT) (Stearns et al. 2001, 662), this terminology was deemed potentially non-ideal for this work. SNOMED CT is not a BFO conformant ontology, and indeed has significant issues with regard to its included layer of upper level terms. Moreover, its size and high degree of specialization mean that it cannot be easily re-aligned without significant time, effort, and input from medical domain experts (Bona, Ceusters 2018) Schulz and Martínez-Costa 2015). Given this challenge a review of ontologies in the NCBO’s Bioportal (Noy et al. 2009) ontology repository, as well as of the OBO Foundry (Edison et al. 2017; Smith et al. 2007) ontologies, was 
conducted to identify possible candidates to replace SNOMED CT. In order for a replacement to be considered, it needed to be BFO conformant, judged easily modifiable to become BFO conformant, or offer significant benefits over SNOMED CT that would justify its replacement. Based on this review however, no other clinical information model having sufficient scope for this application conforms to BFO. Thus, no other information model offered any comparative advantage for implementation of a clinical module.

Given the noted formality issues, only classes from SNOMED CT were included in this work, as SNOMED’s broader information model was deemed largely incompatible with BFO. We note that a recent decision was made to include the Foundational Model Anatomy terms, which are BFO compatible, into SNOMED (Rosse and Mejino 2008). For the moment, however, SNOMED CT content incorporated into IFAMP is restricted to representations of types of things, and loses much of the embedded knowledge concerning relations between these types. Though not ideal, preserving the formality of the larger IFAMP framework was deemed more important than capturing this knowledge.

\subsubsection{Refactoring SNOMED CT}

Four modules (Figure 6) were created from SNOMED CT's class structure using the Refactor tool in Protégé 5.2 (Musen et al. 1995). These included terms describing surgical tools, procedures (specifically surgical actions, which are classified as qualifier values in SNOMED CT), a limited anatomical terminology, and terms relating to clinical roles and environments.

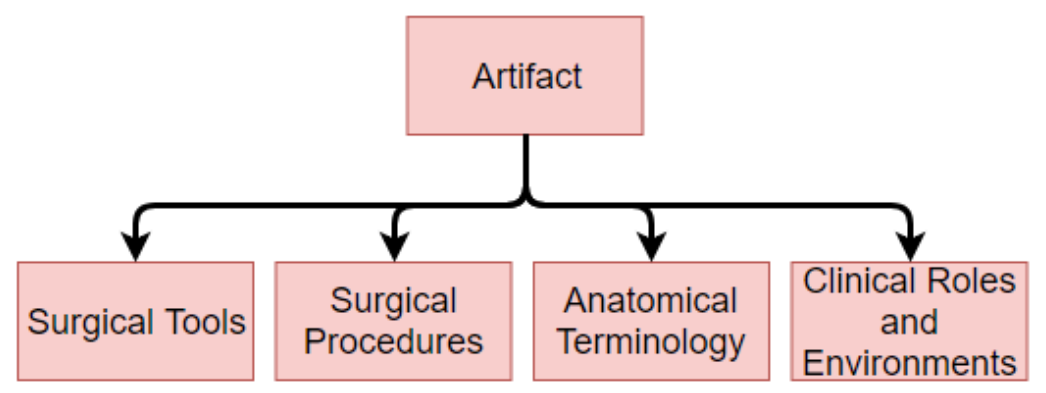

Figure 6. Clinical terminology modules used to define the clinical ontology modules in IFAMP

The tool, procedure, and anatomy modules perform similar functions in the proposed ontological framework. They allow the capture of contextual information about how clinical procedures are performed. When integrated into the larger framework this helps to describe a detailed process for how some new product might be used relative to an existing process. The 
anatomical terminology is included to help in capturing the anatomical context of a given surgical procedure and also offers an ability to express some aspects of user capability in greater detail via interactions with the human factors module of the framework.

The roles and environments modules detail clinically significant aspects of context, such as the types of features an environment - for instance a surgical ward, or a physical exercise room - might have, or the types of individual who might be responsible for various tasks. For example, a designer might be concerned with restrictions present in the environment such as sterile fields, types of resources that might interface with the design, or the types of training people using the designed artifact might be expected to have. Capturing this type of information might allow for better understanding of unspoken stakeholder requirements that emerge in given medical contexts. Within an application these can be refined to allow automated inferences about the design. For example, an application-specific ontology might assert that an object in the sterile field should be sterile. In this more specific application ontology, one can simply define a class that includes all objects residing or planned to reside within the sterile environment. An additional axiom can assert a link to some instance of a requirement specifying certain conditions for sterilizability, packaging, etc. Similar extensions can for example assert requirements for training of various groups, or types of labeling to be used throughout the ontology. While these types of requirements are clearly overspecific for a general medical design framework, in a modular suite of the sort described here their inclusion requires only extension to a more specific set of concerns. Similar approaches can be used to construct application-specific links between domains of any sort where this is found useful.

SNOMED CT’s terminology relating to surgical tools presents problems from an ontologyengineering perspective. SNOMED CT is a large terminology, which classifies various types of surgical tools. However, these classifications are in some places arbitrary, and many classes have multiple parents. They also represent a potentially large ontological commitment as to how various artifacts are to be classified. This means that relevant portions of SNOMED CT cannot simply be imported into IFAMP. Instead, SNOMED CT terms were treated as classifications in a way similar to the approach adopted in the regulation aspect of the framework described above. As a result, it was possible to expand IFAMP's scope to medical devices without the burden of independently developing a medical device ontology. 


\subsubsection{Integrating Medical Terminology}

Other medical terminology content was incorporated into the more general IFAMP framework using several methods. First, the nesting of the BFO and Common Core class hierarchies and information model means that the medical terms first inherit definitions and axioms from their super-classes and may be modified by existing property relations defined in the Common Core Extended Relations Ontology. The clinical terminology was enhanced with additional axioms to provide useful contextual information. First, the existing has part and part of relations are used to construct compound terms represent different sorts of surgical procedures using as starting point 'surgical action (qualifier value)' classes refactored from SNOMED CT. This provides a greater degree of granularity than is typically encountered in SNOMED CT process classes. Other relations were used to define what tools are used during a given procedure, the roles of various process participants (surgeons, technicians, patients, and so forth), and to capture the environmental context of the surgery.

The second integration approach uses functional information to add additional knowledge about various surgical tool and procedures. Since BFO characterizes functions as dispositions that are realized in processes, much of this information was linked directly to surgical procedure classes without an explicit functional model. Instead, the axioms of the process module were expanded with assertions describing the types of function that given processes realize (Figure 7). Information about flows from the Functional Basis Ontology was added in the same way.

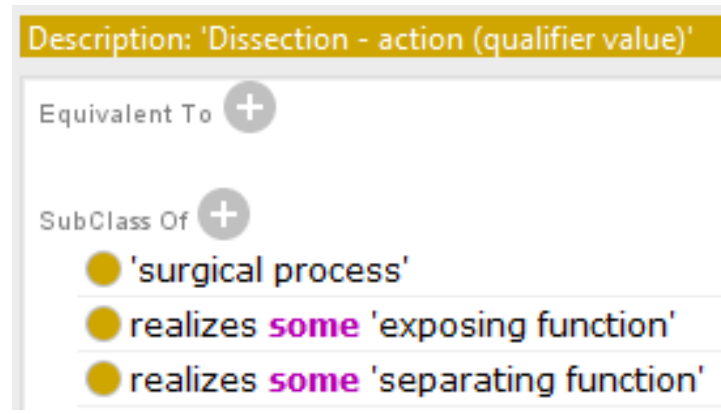

Figure 7. SNOMED CT class definition of 'Dissection' expanded with functional information

A similar approach was used to assert axioms about the medical device classes refactored from SNOMED CT and was similarly applied to regulatory classes. Since the medical device classes are treated as classifications in IFAMP, they are used to classify devices that have some function, again indicated using the functional basis. Thus, a scalpel class from SNOMED CT might 
be asserted to be the classification of an object that has function some 'severing function'. This same logic can be used to indicate virtually any property of a medical device that is permitted to be borne by an object in BFO. Subsequent integration of the medical environment, role, and anatomical terminology modules was then used to support the development of a BFO conformant ontology for human factors design.

\subsubsection{Integrating the Ontologies.}

Co-development of the ontologies using BFO as an upper model, along with careful replication of IRIs where terms are shared between ontologies, means that the integration process was largely a matter of simply importing the ontologies into the framework and allowing them to interact with one another. The order of imports is not important because ontology development software can automatically manage dependences between ontologies. The highly integrated nature of the ontologies ultimately yields the expressive capabilities required by IFAMP.

Where desired, cross-domain reasoning was enabled by importing either single terms or whole ontologies from the relevant domains of interest. However, to enable rapid reasoning, the knowledge bases, rules, and application specialized classes were maintained separately from the completed IFAMP framework. During use, these can be selectively imported along with the relevant terminology from IFAMP to the support these reasoning elements. Thus, while dependencies must be included, not all modules in IFAMP are necessary to access portions of its functionality. This selective approach allows classification and automated inferences about the ontology without the burden of considering potentially unrelated terms, or portions of the framework that are not of interest for an application.

The underlying principle used throughout the development is an independent expansion of expressiveness with extension of the ontology, rather than a re-engineering of dependencies to work with each new domain. This dramatically decreases the effort to ontology development, promotes interoperability, and allows a limited set of patterns to be applied across domains. On their own the top and mid-level ontologies might be used to express very general versions of the information captured in the unified framework. Each domain ontology gives more context to these relations through Is-A type relations between instances and domain classes, or more specific versions of properties as in Figure 8. 


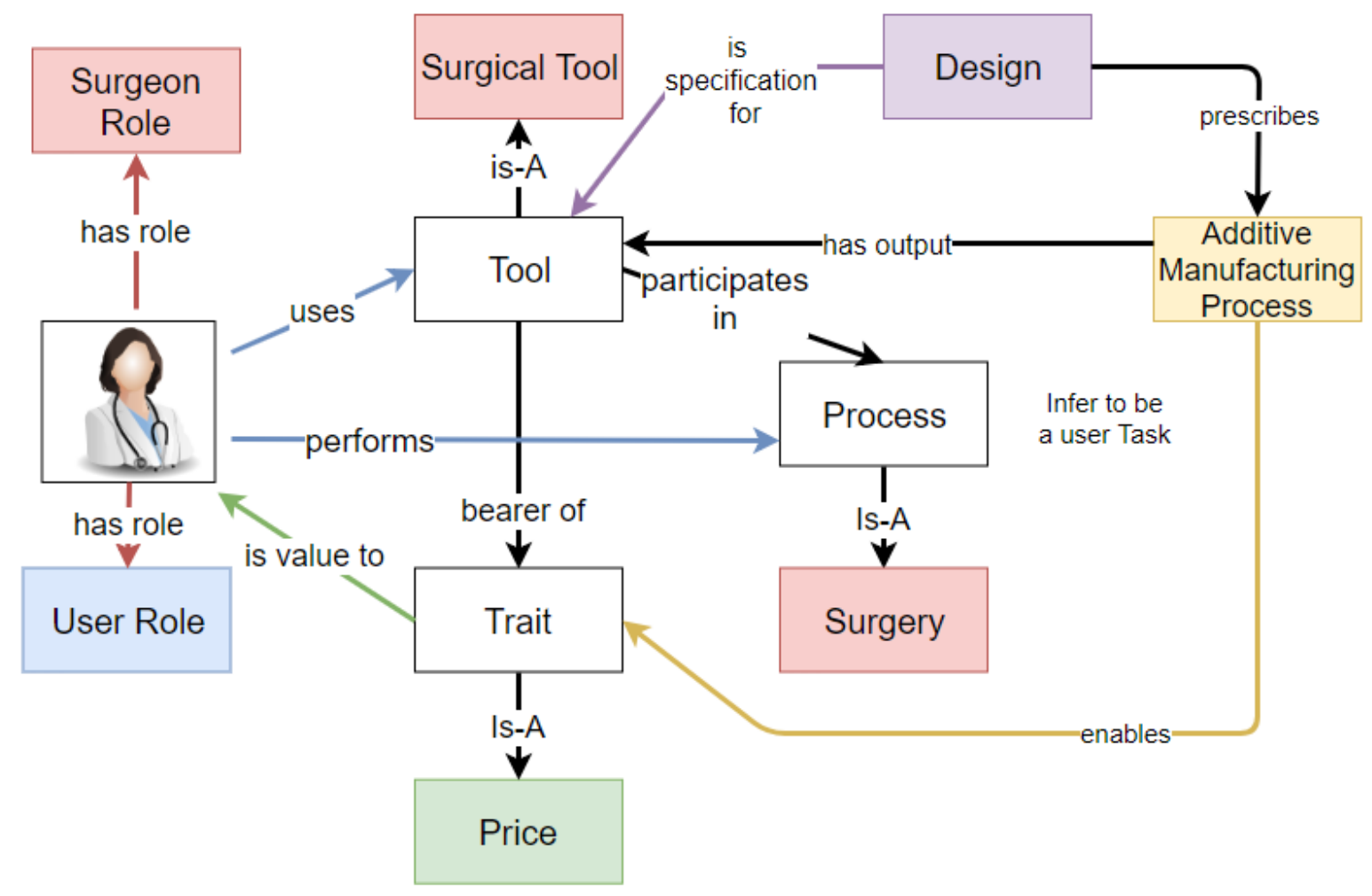

Figure 8. Example of top and mid-level (black and white) expanded with domain modules and used to interconnect domains

In this example, the top and mid-level ontologies provide sufficient terms to say that a person participates in a process, that some object is also involved in that process, and that certain information might be about the object. By adding the stakeholder domain terms, it becomes possible to say the person has the role of a user, and that this person performs the surgical process. The stakeholder domain might also indicate that the person performs this task of interest using a tool. Using the medical terminology, one can more specifically say that the user is a surgeon, the task is a specific type of surgery, and the tool is a specific type of surgical tool. The manufacturing and enterprise modules permit exploration of certain features or traits of the tool that are of value to the surgeon, and potentially how those relate to the way the tool was fabricated. The engineering design module provides the ability to more precisely express relations between design specifications, the product being designed, and its use context. With the addition of each domain it becomes possible to express the same high-level information in more specific ways. 


\subsubsection{Ontology Structure and Domain Relations.}

The overall dependency structure of the ontology is show in Figure 9 below and is discussed in more detail for the remainder of section 2.1. While non-dependent ontologies either do not or only minimally make reference to one another, the shared top and mid-level dependencies of IFAMP allow expressions of common relation types such as participation, aboutness, parthood, and input-output relations. In many instances these mid-level relations can be used to formulate axioms and to construct queries to IFAMP's knowledge base. In the case of domain specific relations, domain and range restrictions are formulated such that they refer to the least restrictive class. For example, a relation between the user of a tool and the tool itself might have its range restricted to a material entity, rather than some term specific to the human factors domain. Similar principles are used to develop class definitions, rules, and other axioms that add greater reasoning capability to the ontology.

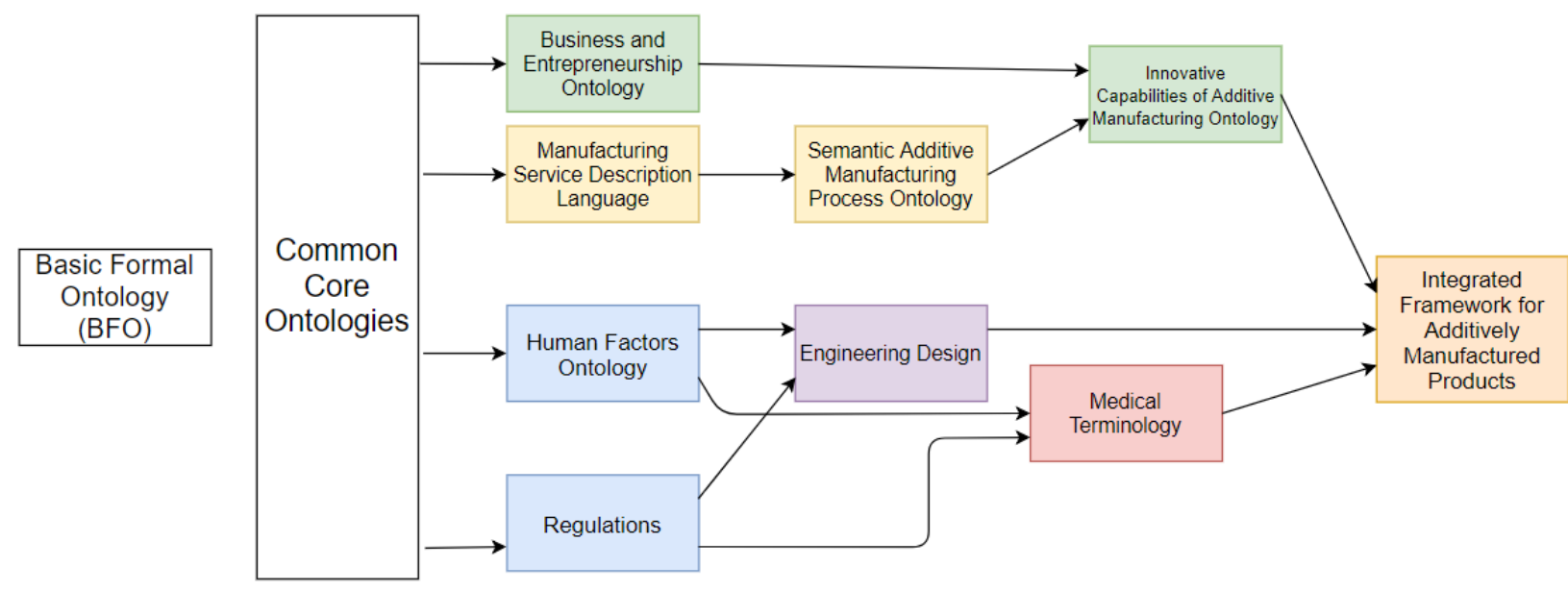

Figure 9. Dependency structure of IFAMP, with each arrow indicating inheritance of some terms from the originating ontology

\subsection{The Design with Additive Manufacturing Method}

On the basis of the above we propose a novel Design with Additive Manufacturing (DWAM) method that aims to utilize the domain and general product knowledge captured in IFAMP (Figure 10). It was hypothesized that the multi-domain approach used in the ontology development would allow detailed knowledge capture and reasoning across domains, most notably in the form of querying. As envisioned, these queries could help to locate useful design information 
that is not directly associated with a given application. This information might then be used to aid in design ideation and issue resolution.

The proposed method aims to integrate domain and additive manufacturing knowledge into the design process, from product conception through design revision and process planning. As envisioned, the knowledge captured in and accessed through IFAMP is used to inform all aspects of the design, to identify possible valuable uses of AM, and to facilitate design ideation, refinement, and selection. Thus, the ontological representation of the design helps facilitate its realization in a finished product. The DQAM method uses a four-phase cycle of need or problem identification, generation of concepts and solutions, design selection, and verification though manufacturing and usability assessment. Each of these is supported by both automated reasoning and queries to IFAMP. If problems or needs are identified, the domain and AM product knowledge base are used to locate either designs that solve similar problems or domain resources that might be utilized to attain some desired function or goal. These are used to inspire design solutions. The resulting solutions are then evaluated using against the stakeholder's preferences in order to select the "best" option. The cycle then repeats until no issues remain and a final design and manufacturing plan are reached. While DWAM is focused on using additive manufacturing, this approach might be extended to conventional manufacturing methods also. 


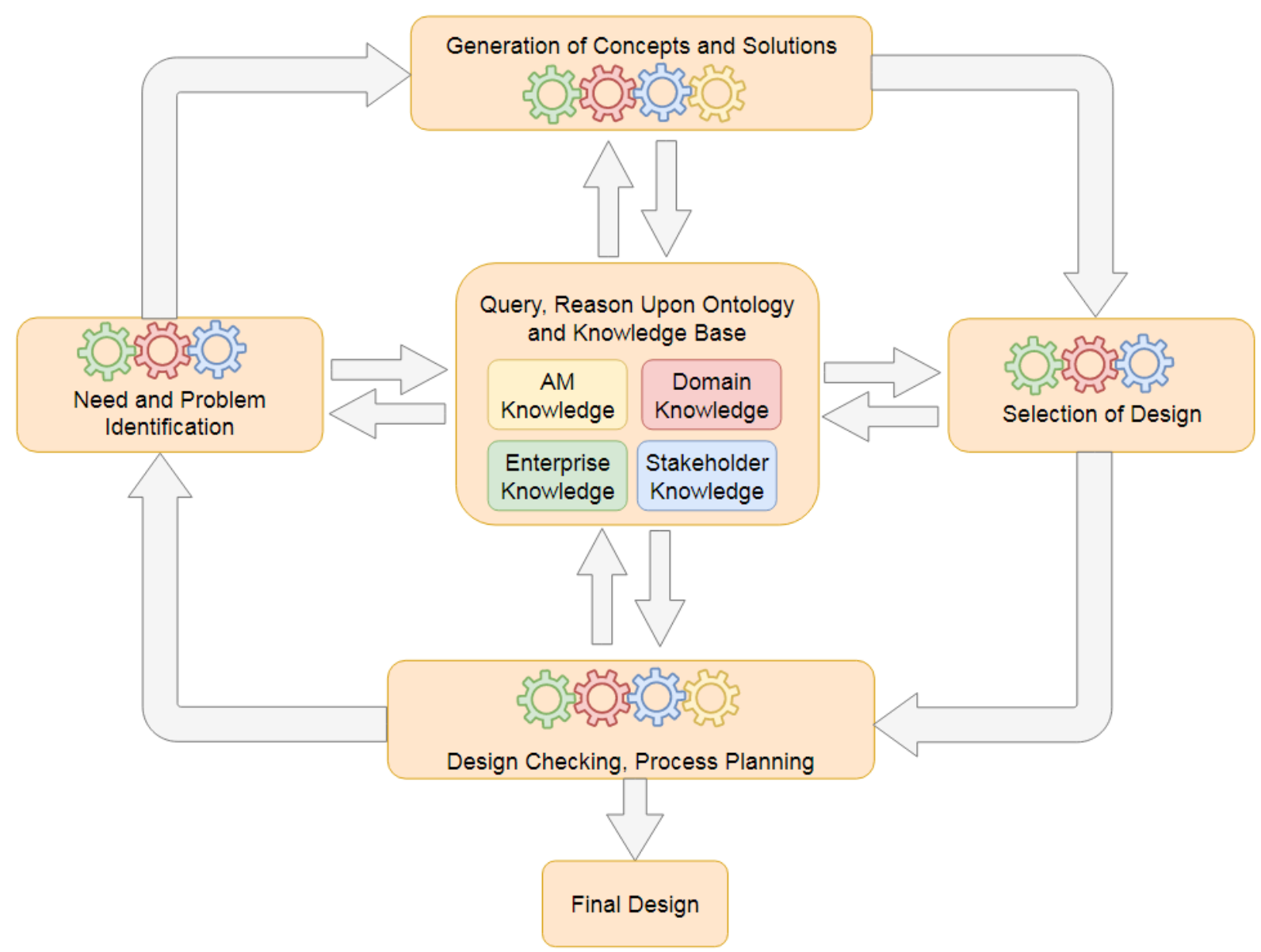

Figure 10. Workflow of the DWAM Method. Gear colors represent knowledge domains depicted in Figure 1 and repeated in the center block. Green indicates market knowledge, blue stakeholder knowledge, yellow manufacturing knowledge, and red domain specific knowledge

The work considered in this manuscript focuses on issues of usability. However, when coupled with the proper knowledge bases and tools, the design checking process envisioned in DWAM might also use automated reasoning to assess manufacturability or to further refine the design (Hagedorn 2018).

\subsection{Case Study}

The utility of the medical device IFAMP framework was assessed using an ongoing undergraduate student design project aimed at introducing a novel new surgical guide for use in total knee replacement surgeries. These require the surfaces of distal femur and proximal tibia to be precisely resurfaced so as to accept and correctly align a joint prosthesis. The student's work focused on market analysis, customer engagement, and concept ideation relating to surgical cutting 
guides. Information gathered in this research process was represented in the IFAMP ontology using the medical domain and human factors modules. IFAMP was further instantiated to support the student's work by adding instances to represent competing devices. The student's design work proceeded in two parts: an initial unaided portion, where they brainstormed and vetted concepts unassisted, and a second portion where they were provided summaries of devices obtained from queries to IFAMP's knowledge base (as described in 2.2).

\section{Results}

\subsection{Ontology Development Results}

IFAMP was successfully constructed from the base ontology modules by importing them into a single ontology file and asserting cross-domain axioms as necessary. Disregarding the knowledge bases, the core framework consists of 2535 classes, 266 properties, and 3915 logical axioms. The AM knowledge bases obtained from ICAM contain 26 products and 15 generic features. Included rules comprised rule sets for five manufacturing processes and 15 heuristic usability rules [53]. Over 150 terms from SNOMED CT including over 60 biomedical device terms were enriched with additional information from other parts domains included in IFAMP. The core ontological framework, sub-modules created to support the case study, and knowledge base were classified using the Pellet Reasoner (Parsia and Sirin 2004) with no inconsistencies.

\subsection{Case Study Results}

\subsubsection{Capture of Total Knee Surgery}

The initial evaluation of IFAMP was based on the case study outlined in 2.3. The evaluation focused on capturing a model of the total knee surgery completed using competing products. A similar instantiation process was used to represent the student's proposed designs.

SNOMED CT term for total knee replacement was used during instantiation of the surgical details in the ontology. Surgical sub-steps were incorporated using the surgical procedure terminology component and linked together using 'has part' and 'part of' relations to create a hierarchical representation of the surgical steps. These are then inferred to be part of the knee replacement operation. The tools used during each step are linked to the main surgical instance (Figure 11). 


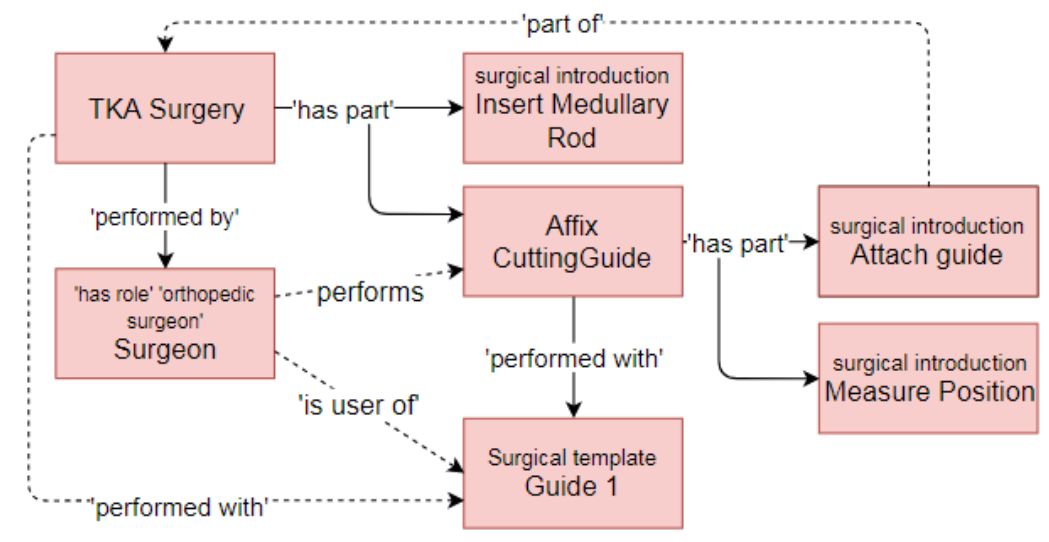

Figure 11. Representation of total knee replacement surgery instantiation. Dotted lines indicate inferred relations

Several other aspects of the surgery can be inferred because surgical terms are linked to the enriched clinical terminology. For example, an instance of some subprocess of the surgery belonging to the 'Surgical removal - action (qualifier value)' class from SNOMED CT's is inferred to realize a 'removing function'. A second axiom reflects the fact that the procedure is performed using an artifact classified as a 'Surgical saw, device (physical object)', from SNOMED CT. The surgical saw classification is similarly enriched with additional axioms reflecting the classified object's 'severing function.' A third instance, this time a directive information entity, specifies that the subprocess occurs in a hospital environment. These relations are inherited by the specific instance of the 'surgical removal - action (qualifier value)' class once the reasoner is synchronized.

The business and human factors modules were used to represent the surgery from a designer perspective. The student found that existing products aimed to improve or ensure the coronal alignment of the knee, reduce the amount of work required, shorten the surgery, and reduce tool use. These potential value contributions were instantiated as opportunities for new products. For example, the surgery realizes some invasiveness that negatively affects someone having the role of the patient during the surgery. Reducing this invasiveness would provide value to this individual (Figure 12). 


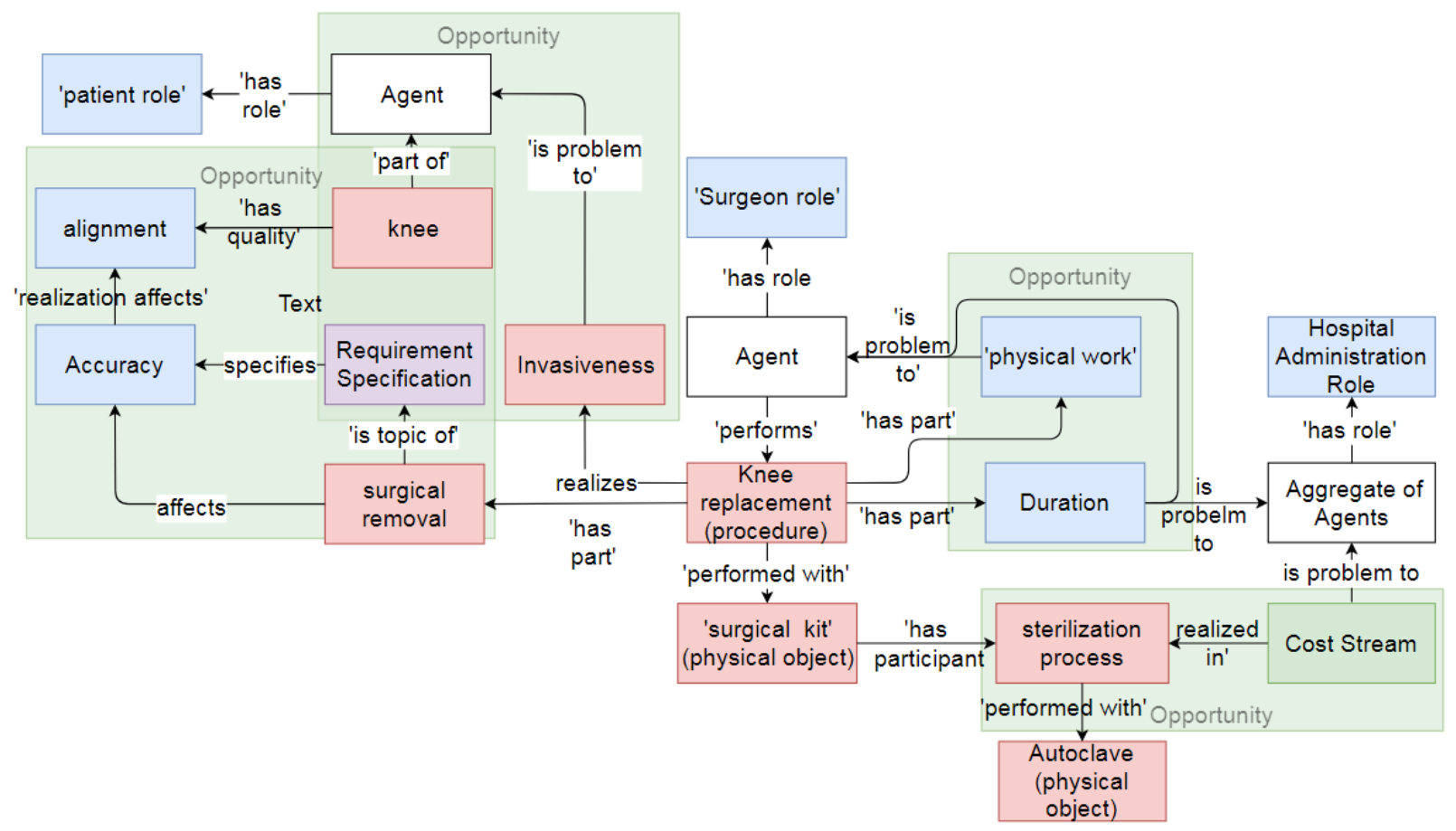

Figure 12. Information model depicting ontology representation of the opportunities for new products targeting total knee replacements

These observations were used to formulate queries to identify possible avenues for new products. They were also used to annotate competitor devices. In both cases the value of competitor devices or students proposed products were instantiated as opportunities. These can serve as the basis for queries to the product knowledge base to identify past methods of pursuing similar opportunities. Thus, the knowledge base grows with each design case. One might also use the representation of the surgery to query for other similar surgical procedures.

\subsubsection{Capture of Stakeholder Information}

The student's stakeholder engagement findings were captured in IFAMP using a combination of its design and medical terminology. The design terminology was used to create specifications or requirements, metrics, and the numerical data included in the ontology. These exist as information entities within the Common Core Ontologies, which in turn characterize or are about other entities. The inclusion of regulatory considerations helped capture information beyond the student's initial product research. In capturing the design specification for the student's work, it was asserted to be a specification for some object that was classified as an orthopedic cutting template in the FDA classification database. The object was also noted as having a 
classification as a 'Surgical template (physical object)' in SNOMED CT. The regulatory classification information links the related requirements and standards to the design and design specification (Figure 13).
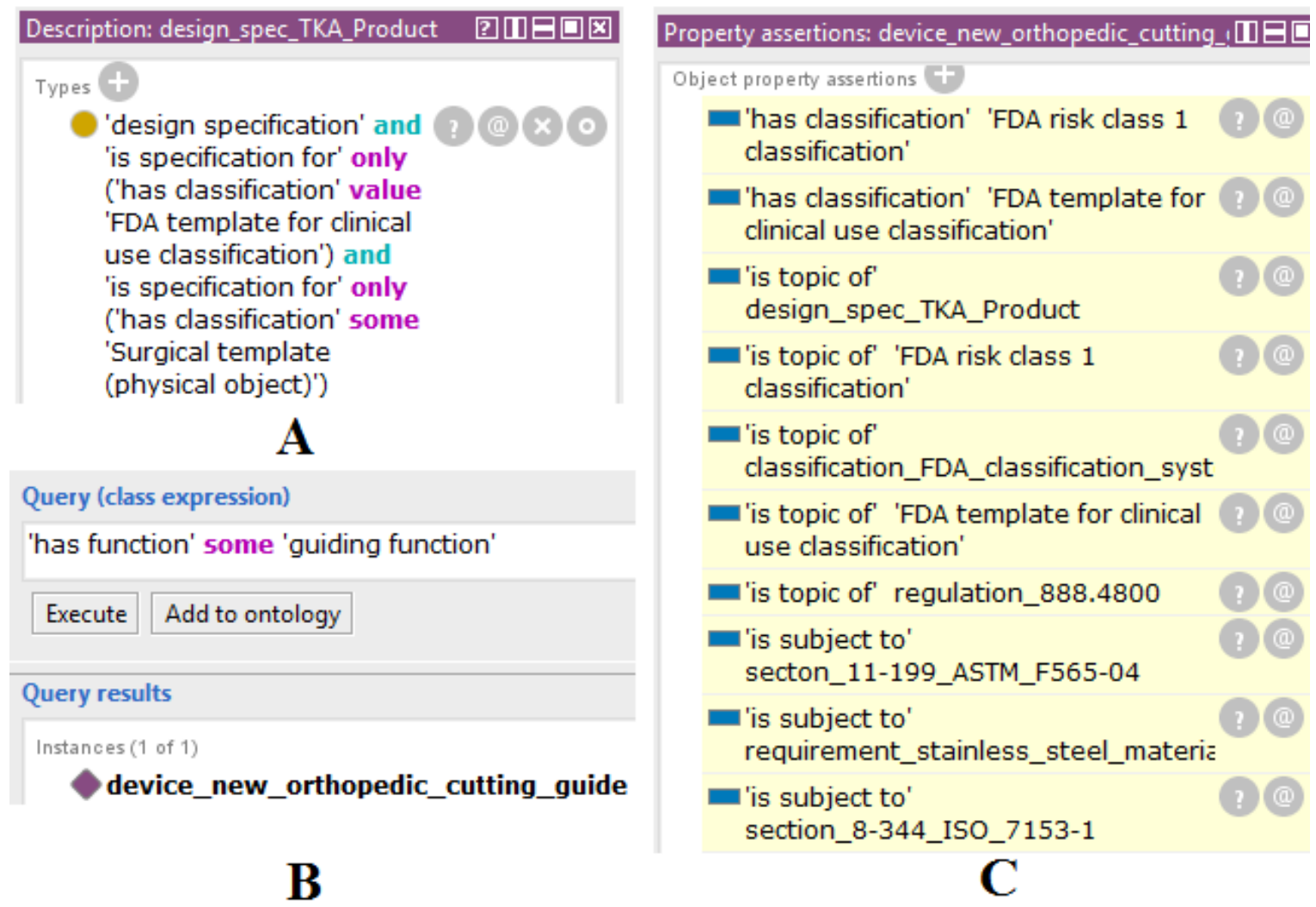

Figure 13. (A) Tbox statements assigning classifications to the design specification (B) Query showing functional information inferred about product from SNOMED CT classification (C) Requirements information inferred from regulatory information

The initial classification used regulatory terminology to link the design to a specific regulatory document, which in turn references standards. Like the surgery capture, the SNOMED CT classification lead the reasoner to infer functional information, this time using regulation and standards models as shown in Figures 3 and 5. In addition to parsing regulatory information, the reasoner also inferred that the student's cutting guide was being designed for a guiding function based on a classification stemming from a SNOMED CT device class.

\subsubsection{Querying for Design Ideation}

The knowledge base of AM products created for ICAM and the newly added clinical domain information were queried to locate potentially related devices that might be used to inspire 
a new design solution. Based on the students work, several avenues were considered. First, functional considerations led to queries for products guiding, aligning, or positioning tools, or those removing material. The opportunities captured while characterizing competitors also led to queries for products used in processes affecting traits such as invasiveness, accuracy, the quantity of tools used, or the duration of some process. These queries returned both classes of surgical tool or processes meeting these conditions as well as instances from the knowledge bases including those reflecting competing products.

\subsubsection{Student Design}

The student researcher created five alternative proposals for knee surgery design over the course of two phases. In the first they brainstormed design ideas without assistance , and in the second they were provided a summary of query results to IFAMP describing 12 broad types of tool or procedure. The student proposals from the unassisted portion of the design focused on adjustments to the procedure aimed at alleviating inconveniences or complexities associated with similar orthopedic devices. The second phase combined features of multiple devices returned by the queries to significantly modify both the process and tooling used to complete the operation. Based on the preference models created from the student's interaction with stakeholders and captured in IFAMP, the student selected one of two variations on this idea for use in a case study with DWAM. Implementation of DWAM

A human factors review was conducted independent of the ontology, and a manufacturability review was conducted aided by the ontology and a set of rules defining manufacturable features for multiple additive manufacturing processes. These follow the form of the rule in section 2.1.3.2 to compare specified feature sizes and dimensions to the limits of various processes. These automated checks flagged several major issues with the student's design. The first is comparatively minor: the guide specified in the design could not be secured prior to fastening with surgical pins. The AM knowledge base was queried to identify instances where some AM feature or product was used to reversibly fix objects in place.. The query returned five instances from the knowledge base (Figure 14). One of these was a surgical clip that used Polyjetting to include a flexible hinge in a single part. . Combined the initial guide design, this might allow the device to snap over the bone. A second returned by the queries was a snap fitting hook. With AM this could be printed directly into the guide and used to tighten the guide around the knee. 


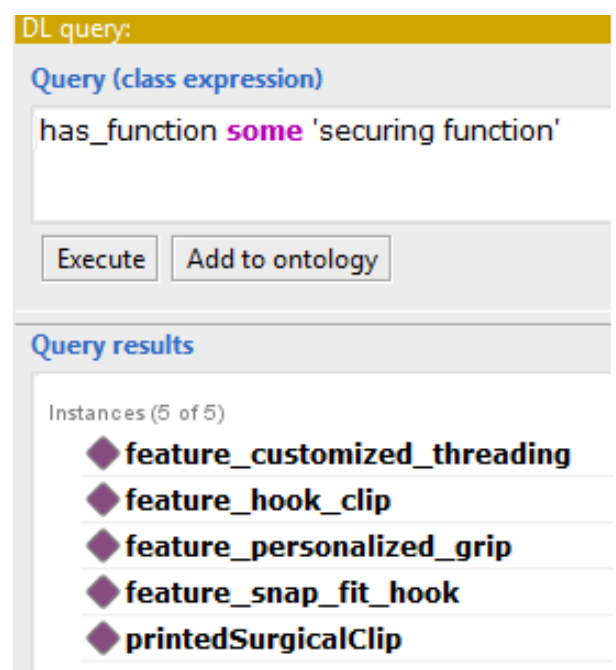

Figure 14. Query results for usability problem fix ideation.

The second issue was control of the tool path, which was deemed insufficient in the original guide. In this case the knowledge base was queried to identify AM products and features that had been used to control motion. This query returned three instances, but two were deemed irrelevant as they dealt with guiding the motion of cable elements. The third was a track and ball feature, which Bin Maidin et al. (Bin Maidin, Campbell, and Pei 2012) noted can be printed in fully encapsulated form using AM. The encapsulation was deemed not useful, but the creation of tracks was considered useful.

The fixation options were evaluated using the preference model derived from the student's stakeholder engagement, with the snap fit ultimately selected. The track option was taken since no other alternatives were identified. The guide was redesigned based on these decisions, and in subsequent review found to be acceptable from a usability standpoint.

\section{Discussion}

The ability to construct IFAMP and, by extension, to execute the DWAM method was entirely dependent on the use of a TLO and careful development principles. Both serve as an instructive demonstration of the advantages of TLOs and the adoption of shared ontology development principles. The development process was made relatively straightforward as BFO is itself very small, and thus makes little or no ontological commitment about anything specific to a domain. Where general terminology was needed, it was possible to draw on existing curated midlevel ontologies such as the Common Core Ontologies. This, along with previous work 
undertaking the realignment of engineering ontologies to a BFO in the form of ICAM, meant that this work could focus on extension of terminology and a knowledge base to new domains and enrichment of that knowledge.

This base information repurposed from existing, published work and the adoption of a welldefined top-level directly facilitated implementation of IFAMP. The inclusion of regulatory and stakeholder information was straightforward as the basic principles governing how different types of entity interact in BFO were already defined. Mid-level ontologies provided a wealth of terms and relations that could be drawn upon and expanded to integrate the user information with a broader design context. Since they followed existing information patterns this process was mostly restricted to defining a core set of human factors domain terms. Even in the case of an existing ontology with formality issues, the existence of published style guidelines helped drive ontology modeling choices towards ones that will not preclude future expansion to new domains. Even if the medical terminologies were inseparable from IFAMP, the treatment of medical terms is such that it need not interfere or overlap unnecessarily with ontologies developed for other domains. Once combined, this development process meant that each module was consistent with the greater whole, and may support subsequent ontology development efforts.

Considering IFAMP in isolation, the underlying hypotheses driving the development of IFAMP and DWAM are all related to the value of linked information. It was hypothesized that the multi-domain approach would allow detailed knowledge capture and reasoning across domains. As seen in the surgical capture, this in fact allowed for a rich view of the surgical process, and from this perspective the ontology was able to capture possible avenues for new devices with potential added value. In a similar fashion, each competitor and design idea could be mapped to a base surgical process, and so the impact of each design on various stakeholders could be fully captured. DWAM was hypothesized to facilitate creative design ideation and problem solving. The ability to obtain relatively useful information via queries of the sort documented throughout this manuscript can be seen as verification that this is the case. The student case study offers some validation also to the information retrieval capabilities of the framework. As hypothesized, surgical methods, tools, and additively manufactured products unrelated to the student's specific design problem were retrieved using general queries to the knowledge base, pointing to a potential educational application for multi-domain ontology initiatives of the sort described. The data 
retrieved through these general queries were in turn relevant and sufficient enough to assist in design refinement and address design concerns raised during evaluations of the student's design.

The student case study points to the practical uses of an ontological approach to support design. Even with a relatively limited set of clinical knowledge, it was possible to express highly detailed descriptions of the clinical context of the knee surgery. This expression was itself helpful in identifying possible avenues for new devices to offer value. On the ideation side, the use of a knowledge base linked to functional, clinical, and value driven terms provided a diverse set of devices that might inspire new design solutions. The multi-domain knowledge in IFAMP also seems to be of note. Much of the student's later designs focus on a change not just to the form of the device, but also to the tooling used. The ideation materials extracted from the ontology included substantially different tools than are used in many of the competing devices. The student noted that this type of information was among the most useful. Greater knowledge of the resources already available proved to be critical to the final design. Aided by domain specific knowledge about types of surgical tool and domain agnostic information about AM features, the student was able to develop a new device concept that differed significantly from their original efforts. This same knowledge was also successfully applied to solve specific design problems identified during further analysis of the design concept.

Despite these promising findings, the case study does leave some avenues less explored. Manufacturability assessment and subsequent query-based resolution of issues would represent a major contribution to the underlying DWAM method. IFAMP and DWAM also do have notable limitations. As with any knowledge-based approach, the quality of knowledge that one can retrieve from the framework depends on what knowledge has already been captured. Formulation of these knowledge bases is difficult and time consuming. Accessing their contents requires a specialized skill set, along with extensive knowledge as to the structure of the ontology itself. Future work should investigate integration of the ontological portions of this work into existing engineering tools and workflows so that they might be made more usable.

Despite these limitations, IFAMP and DWAM have several notable strengths. One aspect that was only touched upon in the case study is the reusability of information from one's own past designs. Notably, the information captured in the ontology for this work might be used as the basis for future design processes. This means that future designs can reuse the insights from the redesign of the student cutting guide. Another strength of the approach is that it enables traceability. 
Instance relations and other ontology constructs capture much of the rationale used to formulate the design, thus problems and solutions to problems can be traced throughout the design process. The effect of specific regulations or stakeholders is similarly transparent thanks to their inclusion in both IFAMP and DWAM. Overall, the results suggest the approach used in IFAMP and DWAM has significant potential to aid in medical device design.

\section{Conclusion}

The mission statement for this special issue notes a number of intellectual challenges and practical limitations standing in the way of useful industrial implementations for ontologies These barriers include: (i) lack of acceptance due to suspicion of ontologies arising from prior failures; (ii) the difficulty for non-experts of reasoning with semantic tools and logics, (iii) ontology development for engineering is seen as overly time-consuming, and finally (iv) problems of implementation arising from the lack of interoperability between ontology models and computeraided design systems. Successful industrial integration requires all four of these barriers to be addressed by researchers and developers in ontological engineering.

The approach taken in this work addresses each of these concerns. The use of a TLO, shared mid-level, and modular domain ontologies developed along similar principles directly help overcome many of the failures of past work outlined both in section 1 of this manuscript and the above barriers. The IFAMP ontologies are interoperable with one another, and reusable alongside virtually any domain ontology conformant to BFO. Indeed, this work takes advantage of existing ontologies developed with similar principles, and demonstrates how even non-conformant domain ontologies such as SNOMED CT might be integrated on an as needed basis. The approachability of semantic reasoning for non-experts is addressed through the introduction of highly repeatable patterns for data representation. These begin in the TLO and mid-level and are extended and made more specific by the domain ontologies developed as part of IFAMP. Semantic inferences are thus more predictable. Very general rules, such as the one used to find design problems in this work, may be used to evaluate similar logic across domains, reducing the need for domain specific development of rules.Non-expert users must still invest effort to understand how entities are linked, but this effort may translate to an entire suite of ontological tools rather than a single, narrowly defined application ontology. 
A major area of focus of this work is to reduce of the effort required for ontology development. This is accomplished through modularity, orthogonality, and reusability. While this work focused on medical applications, those aspects are easily separable from the greater whole. Future ontological development need not redefine the terminology specific to IFAMP's domains, or address more general terms like "capability" that are important across domains. Just as prior ontologies sped the development of IFAMP, so too can IFAMP speed the development of new engineering ontologies. The methods and viewpoint used in this work are also consistent with ongoing ontology development efforts and research into ontology facilitated tool integration and creation of ontology-based engineering tools (Bone et al. 2018).

While not addressed directly in this work, integration with existing computer aided design systems ultimately relies upon a suitably expressive ontological backbone and consistently applicable data representations around which one can construct suitable semantic web technologies. These are both demonstrated in this work, as are some examples of how design tools

and ontology-backed data repositories might be used synergistically for design checking and ideation. Nonetheless, this is an area that requires significant research and development effort. Future work needs to investigate the methodology and architecture required for closer integration between ontologies and engineering software tools as well as. It must also focus on possible avenues by which ontologies may be used to enhance engineering design aides.

\section{References}

Adam, Guido AO and Detmar Zimmer. 2014. "Design for Additive Manufacturing—Element Transitions and Aggregated Structures." CIRP Journal of Manufacturing Science and Technology 7 (1): 20-28.

Ameri, Farhad and Debasish Dutta. 2006. “An Upper Ontology for Manufacturing Service Description.”American Society of Mechanical Engineers.

Aquino Shluzas, Lauren M., Martin Steinert, and Larry J. Leifer. 2011. "Designing to Maximize Value for Multiple Stakeholders: A Challenge to Med-Tech Innovation.”.

Arp, Robert, Barry Smith, and Andrew D. Spear. 2015. Building Ontologies with Basic Formal Ontology MIT Press. 
Barclift, Michael W. and Christopher B. Williams. 2012. "Examining Variability in the Mechanical Properties of Parts Manufactured Via Polyjet Direct 3D Printing.”.

Barrack, R. L., E. L. Ruh, B. M. Williams, A. D. Ford, K. Foreman, and R. M. Nunley. 2012. "Patient Specific Cutting Blocks are Currently of no Proven Value." The Journal of Bone and Joint Surgery. British Volume 94 (11 Suppl A): 95-99.

Berners-Lee, Tim, James Hendler, and Ora Lassila. 2001. “The Semantic Web.” Scientific American 284 (5): 28-37.

Bin Maidin, Shajahan, Ian Campbell, and Eujin Pei. 2012. "Development of a Design Feature Database to Support Design for Additive Manufacturing." Assembly Automation 32 (3): 235-244.

Bona, Jonathan P., and Werner Ceusters. "Mismatches between major subhierarchies and semantic tags in SNOMED CT.” Journal of biomedical informatics 81 (2018): 1-15.

Bone, M., Blackburn, M., Kruse, B., Dzielski, J., Hagedorn, T. and Grosse, I., 2018. Toward an Interoperability and Integration Framework to Enable Digital Thread. Systems, 6(4), p.46.

Bourell, David L., Joseph J. Beaman, Ming C. Leu, and David W. Rosen. 2009. “A Brief History of Additive Manufacturing and the 2009 Roadmap for Additive Manufacturing: Looking Back and Looking Ahead.” Proceedings of RapidTech: 24-25.

Breuker, Joost, André Valente, and Radboud Winkels. 2004. "Legal Ontologies in Knowledge Engineering and Information Management.” Artificial Intelligence and Law 12 (4): 241-277

Chandrasegaran, Senthil K., Karthik Ramani, Ram D. Sriram, Imré Horváth, Alain Bernard, Ramy F. Harik, and Wei Gao. 2013. "The Evolution, Challenges, and Future of Knowledge Representation in Product Design Systems.” Computer-Aided Design 45 (2): 204-228.

Cowell, Lindsay Grey and Barry Smith. 2010. “Infectious Disease Ontology.” In Infectious Disease Informatics, 373-395: Springer. 
Demers-Payette, Olivier, Pascale Lehoux, and Geneviève Daudelin. 2016. "Responsible Research and Innovation: A Productive Model for the Future of Medical Innovation.” Journal of Responsible Innovation 3 (3): 188-208.

Dérand, Per, Lars-Erik Rännar, and Jan-M Hirsch. 2012. “Imaging, Virtual Planning, Design, and Production of Patient-Specific Implants and Clinical Validation in Craniomaxillofacial Surgery." Craniomaxillofacial Trauma and Reconstruction 5 (03): 137-144.

Dinar, Mahmoud and David W. Rosen. 2017. "A Design for Additive Manufacturing Ontology.” Journal of Computing and Information Science in Engineering 17 (2): 021013.

Eddy, Douglas, Sundar Krishnamurty, Ian Grosse, Maxwell Perham, Jack Wileden, and Farhad Ameri. 2015. "Knowledge Management with an Intelligent Tool for Additive Manufacturing.” Boston, MA, ASME.

Eddy, Douglas, Sundar Krishnamurty, Ian Grosse, and Jack Wileden. 2011. "Support of Product Innovation with a Modular Framework for Knowledge Management: A Case Study.” Washington DC, ASME.

Eddy, Douglas, Sundar Krishnamurty, Ian Grosse, Paul Witherell, Jack Wileden, and Kemper Lewis. 2014. "An Integrated Approach to Information Modeling for the Sustainable Design of Products.” Journal of Computing and Information Science in Engineering 14 (2): 021011.

Edison, Ong, Xiang, Zuoshuang, Zhao, Boyang, Liu, Yue, Lin, Yu, Zheng, Jie, Mungall, Chris, Courtot, Melanie, Ruttenberg, Alan, He, Yongqun. (2017). Ontobee: A linked ontology data server to support ontology term dereferencing, linkage, query and integration. Nucleic Acids Research 45(D1), D347-D352.

Farré-Guasch, Elisabet, Jan Wolff, Marco N. Helder, Engelbert AJM Schulten, Tim Forouzanfar, and Jenneke Klein-Nulend. 2015. “Application of Additive Manufacturing in Oral and Maxillofacial Surgery.” Journal of Oral and Maxillofacial Surgery 73 (12): 2408-2418. 
Fenves, Steven Joseph. 2001. Core Product Model for Representing Design Information US Department of Commerce, Technology Administration, National Institute of Standards and Technology.

Fernandes, Rui, Ian Grosse, Sundar Krishnamurty, and Jack Wileden. 2007. "Design and Innovative Methodologies in a Semantic Framework.” American Society of Mechanical Engineers, DETC2007-35446, pp. 237-247.

Fox, Mark S. and Michael Gruninger. 1998. “Enterprise Modeling.” AI Magazine 19 (3): 109.

Furini, Francesco, Rahul Rai, Barry Smith, Giorgio Colombo, and Venkat Krovi. 2016. “Development of a Manufacturing Ontology for Functionally Graded Materials.” ASME 2016 International Design Engineering Technical Conferences and Computers and Information in Engineering Conference, DETC2016-59964, pp. V01BT02A030.

Gardan, Nicolas and Alexandre Schneider. 2015. “Topological Optimization of Internal Patterns and Support in Additive Manufacturing.” Journal of Manufacturing Systems 37: 417-425.

Gibson, I., G. Goenka, R. Narasimhan, and N. Bhat. "Design rules for additive manufacture.” In Solid Freeform Fabrication Symposium, pp. 705-716. Austin, TX, 2010.

Ginsburg, Gill. 2005. "Human Factors Engineering: A Tool for Medical Device Evaluation in Hospital Procurement Decision-Making.” Journal of Biomedical Informatics 38 (3): 213219.

Grosse, Ian R., John M. Milton-benoit, and Jack C. Wileden. 2005. “Ontologies for Supporting Engineering Analysis Models.” Aie Edam 19 (01): 1-18.

Gruber, Thomas R. 1991. "The Role of Common Ontology in Achieving Sharable, Reusable Knowledge Bases.” Kr 91: 601-602.

Gruber, Thomas R. 1993. "A Translation Approach to Portable Ontology Specifications.” Knowledge Acquisition 5 (2): 199-220. 
Gruber, Thomas R. 1995. “Toward Principles for the Design of Ontologies used for Knowledge Sharing?” International Journal of Human-Computer Studies 43 (5): 907-928.

Hagedorn, Thomas J., Ian R. Grosse, and Sundar Krishnamurty. 2015. “A Concept Ideation Framework for Medical Device Design.” Journal of Biomedical Informatics 55: 218-230.

Hagedorn, Thomas J., Sundar Krishnamurty, and Ian R. Grosse. 2016. “An Information Model to Support User-Centered Design of Medical Devices.” Journal of Biomedical Informatics 62: 181-194.

Hagedorn, Thomas J., Sundar Krishnamurty, and Ian R. Grosse. 2018. “A Knowledge-Based Method for Innovative Design for Additive Manufacturing Supported by 19 Modular Ontologies.” Journal of Computing and Information Science in Engineering, in press.

Hagedorn, Thomas J. "Supporting the Design of Additively Manufactured Medical Devices with Knowledge Management through Ontologies.” Dissertation. 2018.

Hirtz, Julie, Robert B. Stone, Daniel A. McAdams, Simon Szykman, and Kristin L. Wood. 2002. "A Functional Basis for Engineering Design: Reconciling and Evolving Previous Efforts." Research in Engineering Design 13 (2): 65-82.

Jardini, André Luiz, Maria Aparecida Larosa, Rubens Maciel Filho, de Carvalho Zavaglia, Cecília Amélia, Luis Fernando Bernardes, Carlos Salles Lambert, Davi Reis Calderoni, and Paulo Kharmandayan. 2014. "Cranial Reconstruction: 3D Biomodel and Custom-Built Implant Created using Additive Manufacturing.” Journal of Cranio-Maxillofacial Surgery 42 (8): 1877-1884.

Kaplan, A. V., D. S. Baim, J. J. Smith, D. A. Feigal, M. Simons, D. Jefferys, T. J. Fogarty, R. E. Kuntz, and M. B. Leon. 2004. "Medical Device Development: From Prototype to Regulatory Approval.” Circulation 109 (25): 3068-3072.

Kumar, Sanjay and J-P Kruth. 2008. "Wear Performance of SLS/SLM Materials.” Advanced Engineering Materials 10 (8): 750-753. 
Kumke, Martin, Hagen Watschke, Peter Hartogh, Ann-Kathrin Bavendiek, and Thomas Vietor. 2017. "Methods and Tools for Identifying and Leveraging Additive Manufacturing Design Potentials.” International Journal on Interactive Design and Manufacturing (IJIDeM): 1-13.

Kumke, Martin, Hagen Watschke, and Thomas Vietor. 2016. “A New Methodological Framework for Design for Additive Manufacturing.” Virtual and Physical Prototyping 11 (1): 3-19.

Laverne, Floriane, Frédéric Segonds, Nabil Anwer, and Marc Le Coq. 2015. “Assembly Based Methods to Support Product Innovation in Design for Additive Manufacturing: An Exploratory Case Study.” Journal of Mechanical Design 137 (12): 121701.

Le, Duc-Hau, and Lan TM Dao. “Annotating diseases using human phenotype ontology improves prediction of disease-associated long non-coding RNAs.” Journal of molecular biology (2018).

Lethaus, Bernd, Lucas Poort, Roland Böckmann, Ralf Smeets, Rene Tolba, and Peter Kessler. 2012. “Additive Manufacturing for Microvascular Reconstruction of the Mandible in 20 Patients.” Journal of Cranio-Maxillofacial Surgery 40 (1): 43-46.

Martelli, Nicolas, Carole Serrano, Hélène van den Brink, Judith Pineau, Patrice Prognon, Isabelle Borget, and Salma El Batti. 2016. “Advantages and Disadvantages of 3-Dimensional Printing in Surgery: A Systematic Review.” Surgery 159 (6): 1485-1500.

Martin, Jennifer L., Daniel J. Clark, Stephen P. Morgan, John A. Crowe, and Elizabeth Murphy. 2012. “A User-Centred Approach to Requirements Elicitation in Medical Device Development: A Case Study from an Industry Perspective.” Applied Ergonomics 43 (1): 184-190.

Martin, Jennifer L., Beverley J. Norris, Elizabeth Murphy, and John A. Crowe. 2008. "Medical Device Development: The Challenge for Ergonomics.” Applied Ergonomics 39 (3): 271283. 
McPherson, Jeffery D., Ian R. Grosse, Sundar Krishnamurty, Jack Wileden, Elizabeth R. Dumont, and Michael A. Berthaume. 2013. "Integrating Biological and Engineering Ontologies.” Portland, Oregon, August 4-7, 2013. doi:10.1115/DETC2013-13527.

Munira Mohd Ali, Rahul Rai, J. Neil Otte, Barry Smith, “A Product Life Cycle Ontology for Additive Manufacturing”, Computers in Industry, 105 (2019) 191-203.

Musen, Mark A., John H. Gennari, Henrik Eriksson, Samson W. Tu, and Aangel R. Puerta. 1995. "PROTEGE-II: Computer Support for Development of Intelligent Systems from Libraries of Components.” Medinfo 8 (Pt 1): 766-770.

Nagel, Robert L., Prem A. Midha, Andrea Tinsley, Robert B. Stone, Daniel A. McAdams, and LH Shu. 2008. "Exploring the use of Functional Models in Biomimetic Conceptual Design.” Journal of Mechanical Design 130: 121102.

Nam, Denis, Patrick A. Maher, Brian J. Rebolledo, Danyal H. Nawabi, Alexander S. McLawhorn, and Andrew D. Pearle. 2013. "Patient Specific Cutting Guides Versus an Imageless, Computer-Assisted Surgery System in Total Knee Arthroplasty.” The Knee 20 (4): 263-267.

Niles, Ian and Adam Pease. 2001. “Towards a Standard Upper Ontology.”ACM.

Noy, N. F., N. H. Shah, P. L. Whetzel, B. Dai, M. Dorf, N. Griffith, C. Jonquet, et al. 2009. "BioPortal: Ontologies and Integrated Data Resources at the Click of a Mouse." Nucleic Acids Research 37 (Web Server issue): W170-3.

Parsia, Bijan and Evren Sirin. 2004. "Pellet: An Owl Dl Reasoner.” Citeseer.

Perez, K. Blake, David S. Anderson, and Kristin L. Wood. 2015. "Crowdsourced Design Principles for Leveraging the Capabilities of Additive Manufacturing.”.

Preece, Daniel, Sarah B. Williams, Richard Lam, and Renate Weller. 2013. “'Let’s Get Physical': Advantages of a Physical Model Over 3D Computer Models and Textbooks in Learning Imaging Anatomy.” Anatomical Sciences Education 6 (4): 216-224. 
Premkumar, Vivek, Sundar Krishnamurty, Jack C. Wileden, and Ian R. Grosse. 2014. “A Semantic Knowledge Management System for Laminated Composites.” Advanced Engineering Informatics 28 (1): 91-101.

Robinson, Peter N. “Deep phenotyping for precision medicine.” Human mutation 33, no. 5 (2012): 777-780.

Rockwell, Justin, Ian R. Grosse, Sundar Krishnamurty, and Jack C. Wileden. 2009. “A Decision Support Ontology for Collaborative Decision Making in Engineering Design. “IEEE.

Rodrigue, Hugo and Mickaël Rivette. 2010. “An Assembly-Level Design for Additive Manufacturing Methodology.” Bordeaux, France.

Rosse, Cornelius and Jose LV Mejino. 2008. “The Foundational Model of Anatomy Ontology.” In Anatomy Ontologies for Bioinformatics, 59-117: Springer.

Ruffo, M., Chris Tuck, and R. Hague. 2006. “Cost Estimation for Rapid Manufacturing-Laser Sintering Production for Low to Medium Volumes.” Proceedings of the Institution of Mechanical Engineers, Part B: Journal of Engineering Manufacture 220 (9): 1417-1427.

Saeema, Ahmed and Storga Mario. 2007. “Engineering Design Ontologies-contrasting an Empirical and a Theoretical Approach.” Guidelines for a Decision Support Method Adapted to NPD Processes.

Schulz, S. and M. Boeker. 2013. “BioTopLite: An Upper Level Ontology for the Life Sciences.”.

Schulz, Stefan and Catalina Martínez-Costa. 2015. "Harmonizing SNOMED CT with BioTopLite: An Exercise in Principled Ontology Alignment.”.

Slover, James D., Harry E. Rubash, Henrik Malchau, and Joseph A. Bosco. 2012. "CostEffectiveness Analysis of Custom Total Knee Cutting Blocks.” The Journal of Arthroplasty 27 (2): 180-185. 
Smith, Barry. 1998. "Basic Concepts of Formal Ontology.” In Formal Ontology in Information Systems, edited by Nicola Guarino, 19: IOS Press.

Smith, Barry, Michael Ashburner, Cornelius Rosse, Jonathan Bard, William Bug, Werner Ceusters, Louis J. Goldberg, Karen Eilbeck, Amelia Ireland, and Christopher J. Mungall. 2007. "The OBO Foundry: Coordinated Evolution of Ontologies to Support Biomedical Data Integration.” Nature Biotechnology 25 (11): 1251-1255.

Smith, Barry and Werner Ceusters. 2015. "Aboutness: Towards Foundations for the Information Artifact Ontology.” CEUR, .

Smith, Barry, Werner Ceusters, Bert Klagges, Jacob Köhler, Anand Kumar, Jane Lomax, Chris Mungall, Fabian Neuhaus, Alan L. Rector, and Cornelius Rosse. 2005. "Relations in Biomedical Ontologies.” Genome Biology 6 (5): R46.

Stearns, Michael Q., Colin Price, Kent A. Spackman, and Amy Y. Wang. 2001. "SNOMED Clinical Terms: Overview of the Development Process and Project Status.” American Medical Informatics Association, .

Štorga, Mario, Mogens Myrup Andreasen, and Dorian Marjanović. 2010. "The Design Ontology: Foundation for the Design Knowledge Exchange and Management.” Journal of Engineering Design 21 (4): 427-454.

Thomas, Daniel. 2009.The Development of Design Rules for Selective Laser Melting.

Wallace, Evan, Kiritsis, Dimitris, Smith, Barry, Will, Chris. "The Industrial Ontologies Foundry Proof-of-Concept Project”, APMS 2018: Advances in Production Management Systems. Smart Manufacturing for Industry 4.0 (IFIP International Conference on Advances in Production Management Systems, vol. 536), New York: Springer, 2018, 402-409.

Waran, Vicknes, Vairavan Narayanan, Ravindran Karuppiah, Sarah LF Owen, and Tipu Aziz. 2014. "Utility of Multimaterial 3D Printers in Creating Models with Pathological Entities to Enhance the Training Experience of Neurosurgeons.” Journal of Neurosurgery 120 (2): 489-492. 
Wohlers, Terry and Tim Gornet. 2011. "History of Additive Manufacturing." Wohlers Report: Additive Manufacturing and 3D Printing State of the Industry Annual Worldwide Progress Report.

Yang, Sheng and Yaoyao Zhao. 2015. “Additive Manufacturing-Enabled Design Theory and Methodology: A Critical Review.” International Journal of Advanced Manufacturing Technology 80. 